\title{
Otros trabajos y otros trabajadores en Barcelona
}

\author{
Julián Porras Bulla \\ Universidad de Barcelona, Barcelona, España. \\ Email: Jporrabu7@alumnes.ub.edu
}

\begin{abstract}
Resumen $^{1}$ : Se describe y analiza los otros trabajos y los otros trabajadores en la ciudad de Barcelona (España) por medio de un caso particular, los chatarreros subsaharianos: reciclador, buzo, cartonero, minador, catador, wastepicker. Partiendo de perspectivas teóricas, de origen latinoamericano, sobre el trabajo informal y la economía popular, sumado a otras teorías más cercanas geográfica y contextualmente, se trata de entender la construcción social de trabajos y grupos de trabajadores no hegemónicos, tejiendo puntos en común y aportaciones cruzadas. Al mismo tiempo, contextualiza los otros trabajos a nivel estructural y evidencia la relación entre formas de trabajo y sujetos. La tarea de estudiar, entender y explicar los otros trabajos y los otros trabajadores en el mundo es importante y urgente, los cambios en el mundo del trabajo, el aumento de los llamados informales y las dinámicas transversales urbanas hacen necesaria la comparación y la complementariedad en el análisis.
\end{abstract} trabajo.

Palabras clave: trabajo informal, chatarreros, Barcelona, sociología del

\section{Others works and others workers in Barcelona}

Abstract: This paper discusses and analyzes the other works and other workers in Barcelona (Spain), through a particular case, sub-Saharan waste pickers: informal recycler, scavenger, chatarrero, reciclador, buzo, cartonero, minador, catador. This research has a theoretical framework based on Latin American perspectives of informality and popular economy, coupled with others geographically and contextually closest theories. At the same time, it contextualizes the other works from structural point of view, and shows the relationship between forms of work and subject. The study, understanding and explaining the other works and other workers are important and urgent, because changes in the world of work, increasing informal jobs and urban transversals dynamics need comparison and comparative frames of analysis.

Keywords: Informal work, waste picker, Barcelona, sociology of work.

\section{Outros trabalhos e outros trabalhadores em Barcelona}

Resumo: Este artigo descreve e analisa os outros trabalhos e os outros trabalhadores na cidade de Barcelona (Espanha) por meio de um caso particular, os catadores de lixo subsaarianos -reciclador, buço, papeleiro, minador, chatarrero, wastepicker-. Partindo de perspectivas teóricas, de origem latino-americano, sobre o trabalho informal e a economia popular, somado a outras teorias mais próximas geográfica e contextualmente, trata-se de entender a construção social de trabalhos 
e grupos de trabalhadores no hegemónicos, produzindo pontos em comum e aportes cruzados. Ao mesmo tempo, contextualiza os outros trabalhos em nível estrutural e evidencia a relação entre formas de trabalho e sujeitos. A tarefa de estudar, entender e explicar os outros trabalhos e os outros trabalhadores no mundo é importante e urgente, pois as mudanças no mundo do trabalho, o aumento dos chamados informais e as dinâmicas transversais urbanas fazem necessária a comparação e a complementariedade na análise. do trabalho.

Palavras-chaves: trabalho informal, catadores de lixo, Barcelona, sociologia

\section{Introducción}

Está claro que el trabajo ya no es lo que era. Flexibilidad, relocalización, desempleo son los ingredientes, aunque no la explicación. Está en marcha un proceso de transformación en el mundo del trabajo desde hace más de tres décadas. Existen múltiples causalidades, cambio tecnológico(Castells, 2000), cambio de era -paso de la modernidad sólida a la modernidad líquida(Bauman, 2004), o explicaciones más estructurales sobre los modos de acumulación (Wallerstein, 2013). Sin embargo, es un proceso complejo que al parecer no entendemos bien.

Enrique De la Garza lo describe como la crisis del mundo del trabajo (2001). Compuesta de varios procesos paralelos: desarrollo tecnológico, disminución del empleo industrial, aumento del empleo en servicios, desplazamiento de la producción a lugares de difícil vinculación entre trabajo y capital, y cambios en las teorías dominantes de interpretación económica. Como consecuencia, la identidad referida al trabajo ha cambiado -debilitamiento de los movimientos laborales y pérdida de importancia frente a otros aspectos de la vida-; acompañado de crisis del trabajo como núcleo de la acumulación capitalista; negación de la teoría valor-trabajo y posicionamiento de la teoría monetarista; sobredimensión del sector financiero, sin contraparte en la economía real; automatización y uso intensivo de capital fijo en la producción industrial; además de procesos de "globalización” y homogenización de la economía mundial y convergencia de modelos productivos.

La principal consecuencia según de la Garza es la pérdida de centralidad del trabajo - de su modelo hegemónico- en la sociedad postindustrial. Que sumado a problemas conceptuales, heredados de los estudios clásicos del trabajo, no permite ver ni las dimensiones, ni las formas del mismo. Frente a este panorama, en sociología y en economía, se ha planteado la discusión sobre el fin del trabajo. Fundamentalmente enfrentados bajo dos argumentos: cada día hay menos trabajo en nuestras sociedades o cada día este se oculta más(Castillo, 1998; Castillo \& de la Garza Toledo, 2000; Pahl, 1991).

En gran medida la explicación sobre el cambio en el mundo del traba- 
jo se debe a que la economía tampoco es lo que era. Parece que cada día existe un mayor alejamiento entre el mundo del trabajo y la economía. Tal vez, como pronosticaba Polanyi (1989) el alejamiento de lo económico del resto de esferas ha acentuado la situación, casi antinatural, de que el trabajo y la tierra sean considerados una mercancía más, sin particulares diferencias frente a un producto intercambiable cualquiera -utilizado de forma instrumental, sin reparar en las consecuencias para los mundos de la vida que esto puede llegar a tener-. Este alejamiento, tal vez, explica el fenómeno de que la mayor parte del capital se produce en el sector financiero, sin relación causal con el mundo del trabajo actual.

La riqueza del mundo en el 2015 según cálculos del Global Wealth Report (Credit Suisse, 2015) era de 250 billones de dólares. Según estos cálculos, la mitad de la riqueza personal estaba en el sector financiero, y al mismo tiempo, se concentraba en un porcentaje parecido en tan solo un 1\% de la población. Si sabemos que la mayor parte de los humanos obtenemos nuestros ingresos por medio del trabajo, no por mecanismos financieros de circulación del dinero, se puede inferir que una parte muy pequeña de la riqueza distribuida se obtiene de los esfuerzos y transformaciones que se llevan a cabo en el planeta tierra. El cada vez mayor alejamiento de la economía real frente a la economía financiera tiene repercusiones locales, concretas y cotidianas en nuestras vidas. Evidentemente para el mundo del trabajo asalariado, pero también está relacionado a todos aquellos trabajos que no hacen parte del modelo hegemónico de trabajo.

Robert Neuwirth lo muestra claramente (2011), en 2009, mil ochocientos millones de personas en el mundo trabajaban en la economía informal y producían cerca de 10 billones de dólares -en este dato no se cuentan los trabajos de las mujeres en el hogar, que aunque harina de otro costal, hace parte de la misma harina-. Si lo vemos desde una perspectiva economicista no es tan grande, sin embargo, si lo vemos como una cuestión social, es una de las características definitorias del mundo actual. Además, evidencia que existen algunas actividades que tienen mayores reconocimientos políticos, económicos y sociales que otras, sin que las explicaciones "productivistas" sean lo suficientemente claras.

El concepto de trabajo informal se puede discutir mucho. No es explicativo, lo informal es altamente heterogéneo, tiene una carga negativa sobre el trabajador, entre muchas más críticas profundas y superficiales. Sin embargo, este concepto evoca, llama la atención, o resalta fenómenos particulares. Existen trabajos y grupos de personas que no entran dentro del modelo hegemónico de trabajo. Y esto, como en otros casos donde se está por fuera de la norma, tiene inconvenientes, genera espacios de subversión, o crea formas paralelas. En este escrito me voy a referir a este tipo de trabajos como los otros trabajos y a los grupos con los que se lo relaciona, como los otros trabajadores. Mostrando que hay dinámicas en las que el determinante es estar por fuera del modelo hegemónico, pero en otras ocasiones, lo determinante es ser otro, pertenecer a un grupo. 
Una de las grandes características, tanto del trabajo informal, como de los otros trabajos y los otros trabajadores, es que se trata de un fenómeno fundamentalmente urbano (Sassen, 1988). Actividades por fuera del modelo hegemónico de trabajo existen en todas las ciudades del mundo. En ocasiones, una actividad se repite en la mayor parte de ciudades del mundo, con mayor similitud, que la que podría compartir con otras actividades de su entorno, y equipararse a otros factores explicativos como la riqueza de la ciudad, la religión del país o la tendencia política del gobierno. Ser vendedor ambulante en Lima, Londres o Barcelona puede parecerse más que ser bombero en estas mismas tres ciudades. Lo mismo se podría decir de ser chatarrero en Taiwán, Praga o Bogotá. Y lo mismo puede suceder con ser músico de calle o bici taxista.

Y Barcelona no es la excepción. En la ciudad existen diferentes actividades que salen del marco hegemónico de trabajo en su mayor parte asociadas a dos características: es una ciudad con una gran industria turística que crea servicios en todos los resquicios de la misma y leyes de extranjería que excluyen sistemáticamente la regularización de ciertos grupos poblacionales. Sumado a otros factores más difíciles de explicar como el racismo general e institucional, tanto público como de los medios de comunicación. Respecto de Barcelona según el Instituto Catalán de Estadística (Idescat) el PIB de la ciudad en 2012 era de 64.241 millones de euros frente a los 1.042.872 millones de euros del PIB español (Instituto Nacional de Estadística). Es decir un 6\% del total de la economía española. Con una tasa de desempleo para el primer semestre de 2016 de $13.3 \%$.

La mayor parte del empleo en la ciudad se da en el sector servicios, según la Encuesta de Población Activa en el tercer trimestre de 2015 el 83\% del trabajo en la ciudad se presentaba en este sector (INE). Respecto del total el 52\% son actividades asociadas a la industria turística de la ciudad (comercio, reparaciones, hotelería, actividades financieras, inmobiliarias, administrativas y servicios auxiliares, actividades deportivas y culturales y otros). Junto a estas actividades reguladas -muchas de estas precariasexisten grupos de actividades que no entran dentro del marco hegemónico de trabajo y que están asociadas al turismo o son colindantes.

Dentro de estas, las actividades más reconocidas en la ciudad son: venta ambulante, chatarreros, músicos de calle y metro, estatuas humanas, vendedores de latas de cerveza, masajistas de playa, prostitución de calle, bici taxistas, repartidores de publicidad, retratistas y pintores de calle, bailarines de calle, afiladores de cuchillos, entre muchas otras actividades que no están dentro del conocimiento público o que sistemáticamente están ocultas. No hay datos sobre los trabajos informales en Barcelona y los datos que existen sobre España tampoco dan mucha información. Según las estimaciones sobre la población ocupada en la economía sumergida en el periodo de 2005 a 2008 variaba entre 4.701.845 y 4.244.639, contabilizados por métodos indirectos como los modelos monetarios o MIMIC (Arrazola, Hevia, Mauleón, \& Sánchez, 2011). Lo único que se puede resaltar de este dato es su tamaño, que podría equivaler al más del 20\% de la PEA de 2008. 
Al mismo tiempo, se puede inferir que desde 2008 la población ocupada en la economía sumergida debe haber ido en aumento, ya que estos datos no recogen el impacto de la crisis económica actual.

Y aunque la información que se tiene sobre el trabajo en la ciudad no dice mucho sobre los trabajadores que están por fuera de los marcos hegemónicos, nos da indicios de que es un fenómeno que sobrepasa las connotaciones superficialmente estéticas de grupos de trabajos marginales, y que más bien, se trata de profundas características de la ciudad, relacionadas a una de sus principales funciones, el turismo.

Pero antes de continuar con el análisis contextual de la ciudad y del caso particular de los chatarreros subsaharianos, es necesario, saber cuáles han sido las teorías que mejor describen, analizan y explican a los otros trabajos y los otros trabajadores.

\section{Sobre los otros trabajos y otros trabajadores}

Descontando por desconocimiento toda la bibliografía asiática y africana, las investigaciones, reflexiones y teorías surgidas en Latinoamérica sobre la economía y el trabajo informal son las que proveen el mayor número de herramientas para interpretar los otros trabajos. El concepto de informalidad se construyó entre la aportación del estudio de 1971 de Keith Hart(1973) sobre el mercado laboral de Ghana y las misiones de la OIT sobre el empleo en Kenia al siguiente año. Desde sus inicios hasta ahora se han generado diferentes enfoques, y ha englobado diferentes aspectos: el trabajo informal, el sector informal y la economía informal. Y aunque sus autores iniciales no relacionaron las actividades informales con características negativas (Chen, 2012), es un concepto que ha estado relacionado a la pobreza.

La palabra informal se refiere a aquello que no tiene una 'forma' adecuada, por esta razón Fernando Mires dice que es un término derivativo y comparativo (1991). La informalidad es un concepto dualista, separa dos grupos que mantienen conexiones para crear un molde aprehensible de la realidad, tratando de comprimir complejidades en pro de lo operacional. No en todos los países tiene una fuerte presencia en el panorama político, como sucede en el caso Español. Sin embargo, es uno de los conceptos clave dentro de toda la terminología de los organismos internacionales sobre temáticas cercanas a condiciones de vida y trabajo-OIT, BM, FMI, ONU-.

La informalidad al tiempo que polimorfa es interpretada desde diversas perspectivas (Lazarte, 2000). Para la OIT (2002), por ejemplo, es una forma económica particular con baja inversión de capital, escasas barreras de acceso y baja utilización de conocimientos y tecnología. Para la perspectiva más cercana al neoliberalismo la informalidad es un fenómeno creado por el Estado al regular actividades y relaciones laborales que entorpecen el 
natural funcionamiento del mercado, impidiendo la acumulación de capital (de Soto, Ghersi, \& Ghibellini, 1987). La perspectiva estructural dice que el sector informal está integrado al sector formal de la economía mundial, en la que esta es una forma de búsqueda de trabajo de bajo costo alrededor del mundo (Portes \& Zhou, 1996; Cimolli, Primi, \& Pugno, 2006).

En la literatura más reciente se puede encontrar un cuarto tipo, digamos mixto, de posición sobre la informalidad, denominado como holístico, ya que busca describir a la informalidad como un fenómeno complejo con muchas variables a estudiar, desde quién la compone hasta las múltiples causas de su origen (Chen, 2012). Según Enrique de la Garza Toledo (2011) existen más de 64 definiciones de informalidad, que se podrían agrupar en tres categorías. Sin embargo, él pone énfasis en que no es importante encontrar la lista de características de la informalidad, sino entender el problema o el fenómeno que se quiere entender y la relación de este concepto con otros. De la Garza dice que la mayor parte de definiciones de informalidad están subordinadas a los datos estadísticos que producen los países, llevando a confusiones entre fenómenos sociales y características legislativas de mercados laborales particulares o sistemas de protección social contributivos.

Desde una visión europea Jensen, Pfau-Effinger y Flaquer (2009) distinguen dentro del trabajo informal tres formas-etapas diferentes. El trabajo de cuidado, el trabajo voluntario y el empleo informal o no declarado. El primero como la primera forma de reivindicación de trabajo no reconocido por parte del feminismo. El segundo en los años 80s puso en relevancia otras formas de producción paralelas u otras formas de desarrollo, dentro de las cuales resaltaban el trabajo voluntario dentro de actividades cooperativas y diferentes formas de trabajos de subsistencia. La última forma que ha sido estudiada es aquella que muestra los trabajos no declarados como una forma de desintegración y problemáticas emergentes a las migraciones, como la marginalización y deterioro de la seguridad social.

La informalidad es concebida casi en la totalidad de los enfoques como un problema, el cual necesita intervenciones para que se disminuya, se elimine o se incorpore (formalización). Al mismo tiempo se puede decir que existe una relación íntima entre las teorías del desarrollo e informalidad(Bodenheimer, 1970). Para comprender el fenómeno total de lo que se ha llamado informalidad no solo hay que conocer las implicaciones teóricas, también es necesario comprender las relaciones entre la política y la teoría. Cuando se habla de la relación entre política e informalidad, se habla de la forma en como bajo este concepto se legitima la organización de los trabajadores que no encajan dentro del molde de trabajo hegemónico.

No sólo existe la influencia política del Banco Mundial, el Fondo Monetario Internacional o la OIT, ciertas interpretaciones de informalidad han sido utilizadas políticamente para respaldar las políticas de desarrollo, por ejemplo, Ronald Reagan y Margaret Thatcher llamaron a los países no desarrollados a adoptar las formulaciones de de Soto como el único camino 
al desarrollo. Se podría decir que la conjunción entre políticas orientadas hacia la microempresa y políticas hacia informalidad se dio en los años ochenta, con varios ingredientes: teorías económicas liberales que relacionan el empleo con el crecimiento económico, la competitividad y esto con la libertad de empresa y el desarrollo económico, y por tanto, el empleo informal con su principal molde, la microempresa (Ramírez Guerrero, 2003).

Para la informalidad, en sus múltiples corrientes, la contraposición formal-informal implica una conexión de sub-alternidad. Martha Chen (2012) plantea eliminar esta sub-alternidad a partir de entender las conexiones entre actividades formales e informales. Sin embargo, conocer las conexiones entre informalidad y formalidad no elimina la crítica de que esta perspectiva dualista, al simplificar, oculta formas de trabajo. Por ejemplo, en el caso español existe una especie de consciencia sobre los trabajos de cuidados y reproductivos, al mismo tiempo, existen amplios grupos invisibles, como pueden ser los vendedores ambulantes, los chatarreros, los lateros, transportistas informales, los artistas de calle, repartidores de publicidad, entre muchas otras actividades.

Sobre la informalidad podemos aprender a olvidar la pretensión de hacer listas de actividades que estén por fuera de un marco legal o de un tipo ideal. Por el contrario, podemos crear grupos de actividades que comparten lógicas, mecanismos de reproducción, o formas de control -como lo explica Enrique de la Garza en sus estudios sobre los trabajos no clásicos, en donde determina que las relaciones de control en actividades realizadas en el espacio público, o en la prestación de servicios, los actores determinantes en la reproducción de la actividad superan el binomio empleadorempleado e incluyen a la policía, los clientes, los transeúntes, etc.-(2011).

Otra lección aprendida es que la informalidad es un indicador de desigualdad. La informalidad es, entre otras cosas, una forma de trasladar trabajo de un grupo de población hacia otro, o de una actividad económica a otra. El ejemplo claro es el del trabajo de cuidados no reconocidos, en donde, una persona descarga las actividades necesarias para su reproducción en otra. La informalidad no sólo estudia el desplazamiento del trabajo, reconoce que las poblaciones que se encuentran en este sector cumplen funciones estructurales, a nivel económico como reguladores del ciclo económico y como presión hacia abajo en el mercado de trabajo; y en el ámbito político como una forma de control social, al ser poblaciones que no se planea integrar, como es el caso de los inmigrantes o tipos de trabajos que no son reconocidos social ni legalmente.

Otra de las ideas útiles desde la lógica de la informalidad es que son actividades que se presentan en todas las naciones (Schneider, 2005), sin que se puedan homogenizar, obedecen a mercados laborales fragmentados y formas de ocupación no reconocidas. Y por lo tanto, se parte de la idea de que son actividades estructurales, y no circunstanciales a los ciclos económicos, características locales, de zonas, o regiones. Ni son exclusivas de sociedades "no desarrolladas", sino más bien, más visibles o políticamente 
determinantes en algunos momentos o contextos. Como ha sucedido en Europa con las crisis económicas.

Un resultado no esperado, no en la teoría que soporta la abstracción que llamamos informalidad sino, en las políticas de formalización, es la poca efectividad que han tenido programas de formalización por medio del soporte a la micro y pequeña empresa. Se puede deducir que la interpretación de los informales como unidades productivas ha llevado a que no se entiendan las relaciones entre los actores, su contexto, las relaciones de cooperación, reciprocidad y control, como tampoco las relaciones que llevan del trabajo a la política, la cultura, el capital y demás instituciones.

Al mismo tiempo, hay dos lecciones que dejan visiones particulares sobre la informalidad. Una es la que plantea Hernando de Soto (1987) sobre el papel fundamental del Estado en el reconocimiento del capital. En su investigación sobre los mercados de vivienda en Lima (Perú) describe una forma de desigualdad frente a las actividades formales. Los resultados de las acciones, intercambios y pactos no son acumulables en forma de capital por parte de los informales, porque como describe el autor, el capital es una abstracción que necesita de la validación de los actores y las instituciones, y son precisamente las instituciones las que no validan estos procesos de acumulación. Totalmente en consonancia con las teorías expuestas por Hart sobre la función de la memoria colectiva en el proceso de acumulación (2000).

En un mundo globalizado donde el capital es un bien común, que se rescata en épocas de crisis, existen poblaciones a las que no se les permite validar. Por ejemplo, los chatarreros sub-saharianos que ocupaban naves industriales en Barcelona han sufrido sistemáticamente este proceso. Los materiales que acumulan y los beneficios económicos que obtienen de su venta no son acumulables, no pueden abrir una cuenta de ahorros, no pueden invertir su dinero en adquirir medios de producción para poder ampliar su forma de trabajo o regularizarla. Y cuando acumulan a través de materiales es una acumulación incierta o precaria, ya que como les ha mostrado la experiencia, en caso de desalojo de los lugares en que trabajan, los materiales que tienen no son ni respetados, ni recompensados.

Volviendo a Hart, el "padre” del concepto, este tuvo una deriva diferente a las otras aportaciones latinoamericanas. Su trabajo posterior ha incluido el análisis del dinero en el capitalismo, y su interpretación ha girado hacia el entendimiento de las instituciones que lo gobiernan, que denomina como arcaicas. La actual posición de Hart es que las actividades informales se mantienen por el poder administrativo de las naciones-estado, que previenen de nuevas formas económicas. Su aportación sobre las otras formas de trabajar es que adjudica un carácter institucional y político al sostenimiento de las profundas desigualdades (2000).

Enzo Mingione tratando de explicar la fragmentación social en Italia, muestra la importancia del tipo de relación social privilegiada, entre las 
típicas del intercambio mercantil, recíprocas o redistributivas dentro de la economía informal (1994). Inspirando en las ideas de Karl Polanyi, sobre su crítica a las relaciones universalistas del mercado como "natural” (1989), Mingione criticando la visión economicista sobre la economía informal y el trabajo informal dice que carece de perspectivas que se centren en las relaciones sociales que allí se construyen.

Para entender el trabajo de los otros y los otros trabajos es necesario aprender de la capacidad política de estos. Esto lo enseñaron bien las teorías sobre la economía popular, bajo la idea de que lo formal y lo informal no están relacionados con las ideas dominantes de antiguo y moderno, sino más bien que lo informal es esencialmente moderno (Moreno, 2001), las teorías de lo popular reivindican la capacidad subversiva de su hacer, y destacan la función productiva y fuente de empleo para el auto sostenimiento(Escobar, 2010; Coraggio, 1998). El exponente actual de este tipo de teorías es Boaventura de Sousa Santos, el cual, lleva años tratando de experimentar con la unión de luchas "postcoloniales" -rurales, por la tierra-, a luchas por el medio ambiente, los movimientos sociales y esos otros trabajadores, o por la creación de otras formas de organización de trabajadores (2011), reivindicando la cercanía entre las teorías de contingencias políticas y sociales, de nuevos movimientos sociales (Castells, 2000) con la suma de las diversas formas de trabajo.

Enrique de la Garza Toledo también resalta esta función en lo que él llama los trabajos no clásicos(2011). La acción colectiva de estos trabajos se cruza cada vez más con los "nuevos" movimientos sociales, mostrando que el trabajo clásico además de perder centralidad en la sociedad también da paso a nuevas formas de crear identidad. Desde otra dimensión, la sociológica, critica a las propias ciencias sociales como coautoras de la construcción de esos otros. Razón por la cual, él estudia el trabajo no-clásico, resaltando que lo que se observa no son trabajos diferentes económica o socialmente, son trabajos que no entran dentro de los modelos hegemónicos de interpretación.

Luego hay autores como Saskia Sassen o Alejandro Portes que han representado toda una tradición de estudios sobre los otros trabajos en Estados Unidos. Estas aproximaciones giran en torno a las relaciones entre trabajos urbanos y migración. Sassen resaltando las características actuales de aumento de la desigualdad, cambios en los patrones de consumo y fragmentación en el mercado de trabajo (1988). Y Portes con su aportación sobre los enclaves económicos(1996). En la que traslada la teoría de Mark Granovetter(1973) sobre la importancia de los lazos débiles en el mercado de trabajo a los trabajos típicamente llevados a cabo por migrantes en Estados Unidos.

Desde otra perspectiva, la del control social, se puede aprender que los otros trabajos están relacionados con poblaciones que son controladas institucionalmente, es decir, esos otros trabajadores. Loic Wacquant muestra como las poblaciones negras en Estados Unidos están inmersas en una 
estructura de control social entre la cárcel, cierto tipo de trabajos y los guetos. Resaltando la idea de que el no reconocimiento de ciertas actividades y ciertos grupos hace parte de estrategias y mecanismos de control urbano, racial y migratorio(2010).

Paralelamente a todo este grupo de teorías sobre los otros trabajos, existen diversos grupos de teorías que enfocan el centro de la atención en que los mundos del trabajo están divididos por distintos grupos sociales. Es así como desde las teorías feministas se ha mostrado que todo el trabajo reproductivo y de cuidado en el mundo es ocultado sistemáticamente por una visión productivista. Al tiempo que el trabajo productivo hecho por mujeres es sistemáticamente menos reconocido (Torns \& Recio, 2012; Burda, Hamermesh, \& Weil, 2013). Lo mismo se puede llegar a afirmar en el caso de los niños y niñas trabajadoras, según los estudios, el hacer de estos otros trabajadores es doblemente perseguido en un sistema económico en el que trabajar no es opcional, ocultando institucionalmente sus contribuciones y precarizando sus condiciones de trabajo (Cussiánovich, 2006). La misma lógica se puede observar desde las investigaciones sobre los sistemas de discriminación, que demuestran como la racialización en el mundo del trabajo repercute en ingresos, reconocimiento y ocultamiento de trabajo (Reskin, 2012).

\section{En Barcelona}

Como ya se dijo en la primera parte, en Barcelona no hay muchos más datos sobre los otros trabajos y los otros trabajadores que los ya expuestos. Lo que se puede es hacer inferencias, deducciones sobre algunas características de la ciudad, y los mecanismos existentes en la construcción social de esos otros.

Por ejemplo, para evidenciar que el mercado de trabajo está bloqueado a los extranjeros no europeos, se puede observar que en diciembre de 2014 el INE calculó que existían más de 195 mil extranjeros no comunitarios con visa de trabajo por cuenta ajena en España y sorprendentemente sólo 6.425 por cuenta propia. Estos datos no corresponden con la realidad de los trabajos que se ejercen en la sociedad, y menos con la de los extranjeros. Si se calcula que en el global de la economía cerca del 17\% de los trabajadores son por cuenta propia, en los extranjeros debería ser por lo menos igual o aún más alto, según bibliografía sobre migración, trabajo y enclaves étnicos(Portes \& Zhou, 1996). Sin embargo, los permisos que concede el Estado para cuenta propia son tan sólo del 3\%. Esto refleja una idea de trabajo deseable, hegemónica y normativa.

Y si de lo general se va a lo particular, podemos observar que en casos específicos como el de los vendedores ambulantes de Barcelona, la prensa construye una imagen delincuencial y de peligro sobre los trabajadores y sobre el oficio. Resalta más los conflictos policiales, que los procesos organizativos, las políticas de la ciudad, o la descripción propia de la actividad. De 156 noticias analizadas que hablan sobre los vendedores 
ambulantes en Barcelona desde mediados de 2015 hasta mediados de 2016 de los principales medios de comunicación catalanes y españoles (El País, La Vanguardia, El Periódico, El Diario y La Directa), 76 de estas trataban sobre temas policiales, en comparación con, por ejemplo, 24 que hablaban sobre política, o 28 noticias sobre el colectivo de vendedores ambulantes.

Sobre los otros trabajos en Barcelona hay una larga historia no escrita, o por lo menos, una historia no divulgada. Los inicios de la actividad de los chatarreros pueden rastrearse desde el siglo XVIII o la venta ambulante en el siglo XIII con el Mercado de Belcaire, hoy atracción turística de la ciudad. La ciudad regula y penaliza constantemente nuevas y viejas actividades. Convierte en otros trabajos a actividades históricamente reconocidas, al tiempo que se responsabiliza a grupos de trabajadores por no hacer las actividades regularizadas. La historia reciente de la ciudad muestra un mismo proceso cíclico de regularización, fragmentación y ocultamiento de las actividades llevadas a cabo en la calle. Primero en el espacio más turístico de la ciudad "las Ramblas”, al prohibir la música, regularizar a estatuas y a vendedores de calle. Esto logró que el número disminuyera a través de una normativa estricta llevó al desplazamiento y desarticulación de los trabajadores.

La constante de regularizar a una pequeña parte de los trabajadores para desarticular la actividad se ha repetido en el caso de los chatarreros, que tras el desahucio de un gran grupo de chatarreros de la nave industrial de la calle Puigcerdà, se crea la cooperativa Alencoop, con la que fue desarticulada y deslegitimada toda una población que se dedica a la actividad, y que no la ha dejado, sino que lo lleva a cabo en forma más oculta.

Lo cual muestra, por lo menos, que las teorías han descrito bastante bien los procesos de control social sobre poblaciones migradas, a partir de esos otros trabajos. Que la persecución, como han sufrido todos los grupos antes mencionados, han sido las infracciones de la Ordenanza de convivencia y civismo de 2006, la cual penaliza todas las actividades llevadas a cabo en la calle, y que dota de un amplio margen de interpretación a la Guardia Urbana para el decomiso de instrumentos musicales, multas y requisas a los vendedores ambulantes y multas a los chatarreros.

\section{El caso de los chatarreros africanos sub-saharianos}

Esta investigación se dio mientras se estaba llevando a cabo el proceso de desahucio de la nave industrial ocupada de la calle Puigcerdà en el barrio de Poblenou -zona de gentrificación de la ciudad-. Esta ocupación era el principal lugar de concentración de chatarreros sub-saharianos en la ciudad. En la nave convivían un gran número de chatarreros de diferentes nacionalidades, aunque, la mayoritaria eran senegaleses. La práctica de ocupar naves vacías por parte de los chatarreros sub-saharianos fue un mecanismo utilizado por décadas en la ciudad (Mendoza, 2014), y finalizó con el desahucio de esta última nave. La actividad y los chatarreros fueron 
desplazados hacia pequeños enclaves, lo que ha derivado en la desarticulación de la comunidad y de sus reclamos políticos. Para esta investigación se realizaron entrevistas a chatarreros en la nave industrial y en el barrio Bogatell, donde se ubican varias bodegas de intermediarios a las que acuden los chatarreros para la venta de materiales.

El trabajo de los chatarreros en Barcelona no se reduce a andar por las calles con un carro recogiendo materiales y objetos. Es un grupo de actividades, de división de funciones, de procesos y conocimientospara seleccionar, separar, transportar, mejorar y vender objetos de re-uso y materiales reciclables.Es una actividad presente en la mayor parte de medianas y grandes ciudades a nivel global, está íntimamente relacionada a la lógica urbana de alto consumo y producción de residuos. Ha sido bien estudiada en los llamados países en vías al “desarrollo” (Samson, 2009), aunque, es necesaria la vinculación con otras realidades y fundamentalmente la interpretación sobre su característica urbana global. En Barcelona existen algunos estudios que ayudan a entender la dinámica general de la actividad (Mendoza, 2014; Porras, 2013), sin embargo, existen muchos agujeros en el conocimiento de las dinámicas de la actividad y de los diferentes grupos que la componen.

El oficio del chatarrero es intensivo en mano de obra, con un alto contenido de azar -los días malos se trabajan largas jornadas y los buenos es posible terminar pronto-. Variables como encontrar una gran fuente de material, la posibilidad de buscar en las horas de recogida de muebles y trastos, las condiciones climáticas, o la escasez de material en una zona, determinan el recorrido diario. Esuna actividad constante de búsqueda, no es lineal de un punto a otro, incluye acercarse a los contenedores, a las zonas donde se han dejado materiales, entrar, esperar, preguntar en construcciones.

Según los cálculos realizados con la información suministrada, los chatarreros son altamente activos. Si en promedio caminan 17 kilómetros esto equivale a 24.285 pasos (siendo $70 \mathrm{~cm}$ la distancia de un paso promedio), según estudios sobre el deporte y la actividad física un adulto que supere los 12.500 pasos por día es una persona altamente activa (Lopez Chicharro \& Fernández Vaquero, 2006). Si a esto se suma el hecho de que en promedio el gasto energético por hora de caminata es de 180 calorías (De Lucio, 2004), más el gasto calórico de las funciones básicas del cuerpo es de 1200 calorías, el consumo es de 3000 calorías diarias. La teoría simplificada sobre el gasto energético dice que la cantidad de calorías gastadas tienen que corresponder con la cantidad consumida, en el caso de los chatarreros, y según los datos suministrados por algunos de ellos, el dinero destino a alimentación es inferior a 5 euros diarios.

El trabajo de la mayoría de los chatarreros subsaharianos no permite el ahorro, ni excedentes para poder enviar a sus familias. El dinero ganado diariamente alcanza para la reproducción simple de su forma de trabajo: alojamiento, comida y transporte - para aquellos que viven en el extrarradio.Esta situación según reportan se ha acentuado con la crisis, ya que hay 
menor producción de residuos, y aumento en la competencia por los materiales, de personas que trabajaban en construcción o en otros sectores y se desplazaron hacia el reciclaje, evidenciando los enclaves étnicos (Portes \& Zhou, 1996), en la forma en la introducción a la actividadpor parte de un amigo o conocido sobre materiales, modos de transporte, lugares de venta y precios.

Las actividades recreativas, según los chatarreros entrevistados, son escasas. En primera medida por no tener ingresos suficientes para esto, sumado al aislamiento que provoca la actividad. La población de chatarreros subsaharianos es fundamentalmente masculina, en las observaciones no se encontró a ninguna mujer realizando la actividad en la calle, a diferencia de otros colectivos como los rumanos o los gitanos, en donde muchas mujeres o parejas recogen materiales.En el caso de la nave de Puigcerdà la situación era similar, aunque el pequeño grupo de mujeres que estaba en la nave se dedicaba fundamentalmente a actividades reproductivas.

\section{La construcción social de la actividad}

Respecto a las demás relaciones sociales, existe una gran diferencia entre los chatarreros de la nave de Puigcerdà y los del barrio de Bogatell. La construcción de solidaridad y respaldo es relativamente sólida en la nave, ejemplificado también en los eventos y protestas que han llevado a cabo para no ser desalojados. Igualmente después del desalojo en los procesos de asesoramiento legal, que acompañados de organizaciones sociales muestran cohesión interna, sumado al capital social construido para llevar a cabo las actividades económicas.

Caso contrario se presenta con los chatarreros de Bogatell, en donde la interacción social se da por cercanía del lugar de residencia -en el caso de aquellos que viven en el extrarradio muchos están ubicados en las mismas ciudades y barrios- , por empatía en los recorridos por la ciudad, y en los momentos que venden sus materiales en las bodegas.

Las relaciones que los chatarreros establecen con los demás son en general buenas. Es muy diciente el caso de respaldo de la Junta de vecinos del barrio de Poblenou en contra del proceso legal de desalojo de la nave de Puigcerdà. Lo mismo sucede con los chatarreros de Bogatell. Existen casos de racismo pero fundamentalmente los chatarreros los asocian a eventos aislados.

Respecto de la relación con la policía los chatarreros afirman que es buena y respetuosa. Sin embargo, tienen la sensación dentro de la nave que entran policías en secreto para controlar las actividades que llevan a cabo. Igualmente, cíclicamente existe alguna presión por los carros que utilizan los chatarreros, ya que en su mayoría son carritos de compra de supermercados. En general, la presión policial en Barcelona no está enfocada sobre los chatarreros, o en la práctica de su trabajo. El mayor control se realiza sobre las ocupaciones que tienen y las bodegas donde venden. 
Lo mismo sucede con la relación que se establece con el intermediario. Es una relación de cooperación en primera medida, pero también de confianza, y de dependencia relativa. El intermediario es el bodeguero, el eslabón entre la economía formal y el trabajo informal de los chatarreros. Este se dedica a ordenar, separar, mejorar algunos materiales y transporte hacia los lugares de venta. La figura del intermediario ha sido bastante estudiada, tanto para actividades formales como informales. Como pieza fundamental que organiza mercados, tiene una posición, medios o información privilegiada, y por lo tanto, puede ser un facilitador, pero al mismo tiempo, ocultar formas de explotación, corrupción, procesos de acumulación o aumento de desigualdades(Stovel \& Shaw, 2012). En actividades económicas informales, como lo describe Ubaldo Martínez Veiga (1989), puede llegar a ser el centro de una actividad en la que se concentra trabajo intensivo poco productivo, como puede suceder con algunas prácticas de chatarreros, en las que tras largas jornadas de trabajo se consigue poco material de difícil clasificación y separación, lo que como resultado tiene un producto de valor inferior al costo de su producción.

Por otro lado, la imagen que se construye del chatarrero,como trabajador, no es tan buena. Ni para los chatarreros, ni la imagen que ellos creen que tienen en el resto de la población. Los mismos chatarreros no reconocen que la actividad que ejecutan sea un trabajo, lo relacionan con una actividad deshonrosa o circunstancial. Esto se constata con la imagen que tienen de los otros colectivos de chatarreros y sobre la imagen que tiene el resto de la población sobre el trabajo. Algunos chatarreros subsaharianos entrevistados, al preguntarles sobre las razones de por qué otros colectivos trabajaban con la chatarra, aducen que lo hacen por características culturales, y no por las condiciones estructurales.

Existe una idea constante sobre la actividad: esto es una situación transitoria. El proceso de ocultamiento del trabajo de los chatarreros está construido socialmente, y se da también entre los propios chatarreros. Y aunque, sea una actividad económica con todas los ingredientes de cualquier otra, esta se resignifica bajo la idea de labor -más cercana a la idea sobre actividades necesarias para la supervivencia, o para la reproducción simple, que a la idea de un trabajo transformador- (Arendt, 2009).

La idea de los chatarreros como otros trabajos y otros trabajadores permite entender lo perverso que es el modelo interpretativo del mercado de trabajo como un escenario donde sólo pueden participar aquellos que son reconocidos de antemano como válidos.

\section{Mecanismos y estrategias de ocultamiento del trabajo}

Buscar el trabajo oculto, o los mecanismos que lo ocultan, es un trabajo por entender cómo la sociedad como un todo lleva a cabo sus prácticas económicas. Al mismo tiempo es necesario pensar el trabajo desde una perspectiva humanista o amplia, en la que el trabajo es una condición 
humana, antropológica, que configura símbolos, sentidos, relaciones y realidades (Reichman, Madorrán, \& Echavarría, 2013).

Si se observa desde la perspectiva económica de la oferta y la demanda, los chatarreros son proveedores de materia prima para algunas actividades productivas. Pero, si se trata de analizar la función social de su trabajo se observan algunas diferencias. El sistema de recolección de residuos sólidos en Barcelona fomenta la separación de materiales en la fuente, con lo cual, existe un programa de aprovechamiento de estos residuos. Se supone que si este funciona en forma exitosa el trabajo de los chatarreros se presenta como una especie de sobre-trabajo, en donde estos recolectan los materiales sólo como una forma de subsistir en una economía con alto desempleo y segmentada hacia los migrantes. Por lo que se podría deducir que es un trabajo intenso en mano de obra improductividad.

Por otro lado, no se puede negar que existe toda una estructura económica que se mueve alrededor de materiales reciclados, que sale de la lógica del manejo de residuos de la ciudad. Esta estructura sostiene y al mismo tiempo fomenta el trabajo de los chatarreros. Algunas de sus actividades no están limitadas a las lógicas económicas; difíciles de explicar desde la razón de costo-beneficio. Por ejemplo, una parte de las actividades que hacen los chatarreros es sacar los excedentes de materiales en las construcciones o reformas en Barcelona,desplazando trabajo del constructor al chatarrero. El otro caso, en el que recolectan objetos de re-uso, estos objetos al final tienen dos funciones: recuperar una parte de valor de objetos con valor de cambio y de uso, y disminuir el costo de compra de artículos necesarios para una parte de la población. Es de nuevo un desplazamiento de trabajo del comprador al chatarrero. En ninguno de los dos casos productivo, pero inherente a las actividades del sistema capitalista.

Es una discusión que parte del supuesto que las actividades productivas y reproductivas son constitutivas del mismo continuo económico, y así no sean trabajos que incrementen la circulación de riqueza en la sociedad, son actividades que desplazan labor de un grupo de población a otro. Teóricamente se pueden establecer definiciones delimitadas y claras sobre el trabajo, la duda surge sobre la construcción social del trabajo. Respecto de este tema es difícil llegar a una conclusión total, sobretodo porque el lenguaje que usamos en forma cotidiana es flexible a muchos contextos. Por ejemplo, la mayor parte de los chatarreros entrevistados piensa que el suyo no es un trabajo, sin embargo, no dudan que sea una actividad productiva. Dudan de la legitimidad de la actividad por sus componentes normativos -lo que debería ser un trabajo-. Pero no dudan que haga parte de una lógica económica rentable y que genere excedentes.

\section{El reconocimiento social de la actividad}

Si se observa la forma en cómo tratan los medios de comunicación la actividad que realizan los chatarreros, existe una especie de concepción cercana al trabajo de supervivencia, ligada a la idea de trabajo reproductivo, 
sin mayor impacto económico. Y por lo tanto, se construye una imagen de una población en condiciones precarias, pasando a segundo plano el aspecto laboral.

Esto mismo se observa en el tratamiento que recibe la comunidad por parte de Carla Martínez Castro, Magistrada Juez del Juzgado de Primera Instancia Número 21, en el juicio de desahucio por precario contra los habitantes de la nave de la calle de Puigcerdà.La magistrada describe a la población de la nave por una condición circunstancial que no es comprobable, ser personas sin recursos. Describe el uso de la nave en forma imprecisa, como si su actividad se limitara a la recogida y venta, obviando las estructuras sociales necesarias para este trabajo, que están asociadas al uso de la nave. Contrario al discurso de los chatarreros, para estos la nave tiene como principal función ser un medio de producción.

Si se adoptan las descripciones de los medios de comunicación y de la sentencia por desalojo como una expresión social de la actividad económica, se puede observar que la imagen socialmente construida de los chatarreros es de una población pobre, con un trabajo precario, en donde su actividad no tiene ninguna función social, y se considera como un problema. Esta construcción oculta las relaciones entre el capital y el trabajo, no hay ninguna mención acerca de las empresas o industrias que compran o usan el trabajo de los chatarreros, más aún, no existe ninguna referencia a la capacidad económica de los chatarreros, como si fuesen económicamente inactivos. Esta imagen se contrapone a la idea que tienen los chatarreros sobre su función social, donde se recalca su potencial de trabajador y la necesidad de independencia económica por medio del trabajo.

\section{Las políticas sociales y el ocultamiento del trabajo}

La migración de subsaharianos a Barcelona ha tenido un aumento sostenido desde hace más de una década. Si se toma a la población senegalesa como ejemplo, esta ha pasado de 241 inscritos en el padrón municipal de Barcelona en el año 2001 a 1.112 en el año 2012 (Departament d'Estadistique, Ajuntament de Barcelona)). Según la información recolectada, una de las problemáticas a las que se enfrenta la población chatarrera es que su tratamiento ha sido siempre el mismo, ayuda humanitaria para poblaciones vulnerables. Sin deje de ser paradójico que se tomen políticas de urgencia para poblaciones que forman parte de problemáticas generales y estructurales.

No existen políticas sociales particulares para los chatarreros en Barcelona. De hecho, los entrevistados en el barrio de Bogatell reportaban un alejamiento institucional casi total. En cierta medida esta ausencia de políticas la ven como una expresión de libertad frente a otros países y ciudades. En el caso de los chatarreros de la calle Puigcerdá estos accedían fundamentalmente a atención humanitaria, reubicación de la población de la nave -llamado eufemísticamente alojamiento temporal paliativo-, y programas de inserción socio-laboral. Paralelo a esto surgen otras actividades 
o compromisos como los adquiridos por el Ayuntamiento en el desalojo de la nave: como un programa de regulación respecto de su situación migratoria -compromiso de dar un concepto favorable en la solicitud de permiso de residencia por arraigo social, y agilización de los trámites institucionales-, así como jornadas en conjunto con las embajadas de países africanos para refrendar pasaportes y llevar a cabo políticas de retorno voluntario.

En el 2013 la situación estaba mediada por la Cruz Roja como entidad imparcial. Sin embargo, la comunidad relacionó directamente sus actividades como formas para desintegrar la comunidad. En esta crítica al papel de la Cruz Roja se toca un tema fundamental, una organización que normalmente se dedica a actividades urgentes, ¿por qué se dedica a implementar políticas sociales que se supone deberían ser sostenidas en el tiempo y consistentes? Esto muestra un síntoma del ocultamiento del trabajo por parte de las políticas sociales, tratar a los chatarreros en una dimensión coyuntural invisibiliza su carácter como trabajadores, como constructores de valor y de su función social.

La legislación sobre la residencia, y la necesidad de tener un contrato para obtenerla, influye en el reconocimiento de los otros trabajos como trabajos de mentiras, tanto legalmente como para la auto-comprensión. No hay permiso de trabajo para los trabajadores informales, así estos lleven décadas en este oficio, la legislación no les permite ascender laboralmente, ni especializarse. Es un sistema migratorio que excluye cualquier tipo de regularización.Aunque las políticas sociales no incluyen las políticas migratorias de un país, son lógicas muy cercanas. La ausencia de reconocimiento legal lleva, no sólo a que no se pueda buscar nuevas formas de trabajo, sino que invalida el trabajo del chatarrero a través de las diferentes formas de expresar y consolidar el trabajo, como la posibilidad de arrendar una bodega para almacenar, o adquirir nuevos medios de producción o transporte.

Por lo tanto, se puede observar cómo se consolida el ocultamiento de un trabajo determinado. Es un sistema auto-referencial, el no reconocimiento institucional influye en la imagen socialmente construida, y ambas en el valor que socialmente tiene el trabajo. Institucionalmente no se reconocen realidades por fuera de las humanitarias para los chatarreros en Barcelona. Si se reconocieran las realidades laborales de los chatarreros sería necesario reconocer su función social, legitimando no sólo a la actividad sino a sus ejecutores.

\section{El capital como forma de representación del trabajo}

La otra forma de ocultamiento del trabajo se refiere al no reconocimiento del capital, como expresión de los frutos y la materialización del mismo.El capital es el modo en como se expresa el excedente del trabajo. Por lo tanto, teóricamente se puede pensar como el sobrante del valor creado en un trabajo. Es esa parte del valor creado que se acumula, luego de reproducir las condiciones iniciales de la actividad, para poderla ampliarla y acumular en mayor medida. Está claro que el capital tiene que tener un respaldo 
institucional, y este respaldo no es, al final de cuentas, sino una forma de reconocimiento del trabajo necesario para poderlo acumular (de Soto, Ghersi, \& Ghibellini, 1987).

En el caso de los chatarreros subsaharianos en Barcelona es posible ver ausencias de respaldo a los capitales que estos han construido. Además de reglas que dificultan su participación en el proceso de acumulación. Existe toda una discusión sobre si las pequeñas unidades productivas cumplen los requisitos para ser denominados como capitalistas, que su lógica sea acumular capital. En algunas interpretaciones sobre el auto-empleo y la pequeña empresa se dice que estas son trabajo externalizado donde se borra la relación entre capital y trabajo, y por lo tanto estas actividades no concentrarían capital, las que lo concentrarían serían las empresas grandes para las cuales trabajan. En el caso de los chatarreros es innegable que existen unas condiciones necesarias para reproducir la actividad. Y aunque no se pueda afirmar categóricamente que juegan el rol de acumuladores capitalistas, la necesidad de bienes, medios de producción o dinero para llevar a cabo la actividad, se puede interpretar como el capital necesario.

En el caso de los chatarreros existen dos ejemplos dicientes sobre la ausencia de respaldo institucional a la hora de acumular. El primero sucedió tras el desalojo de la nave de Puigcerdà, todo el capital que los chatarreros acumularon allí era legítimamente de ellos, no fueron reconocidos los materiales acumulados, los mejoramientos a la nave para la producción, las estructuras sociales de producción y todo el prestigio como un lugar donde se vendían materiales reciclables y objetos de re-uso. Las medidas adoptadas en el desalojo no salvaguardaron el capital que los chatarreros tenían allí. Si se observa desde la perspectiva de la producción nacional, el capital en una de sus facetas es un bien común a toda la sociedad, y una de las funciones de los Estados es tratar de salvaguardarlo. Para el caso de estos chatarreros no fue así.

El otro ejemplo, es el de la imposibilidad de validar las inversiones de capital en medios de producción. La posibilidad de escalar económicamente dentro de la actividad necesita de una inversión, puede ser en el alquiler de una bodega, o en la compra de un coche para poder recoger y llevar los materiales a mejores mercados o compradores. Sin embargo, esta posibilidad está mediada por la situación legal de la residencia. Sin un permiso de residencia es imposible lograr un mejoramiento dentro de la actividad. Esta barrera institucional lleva a la reproducción de la actividad en una forma menor que simple.

\section{Conclusiones}

Como actividades transversales en todo el mundo, es necesario conocer e interpretar mejor los mecanismos y estrategias de reproducción de las mismas. Los otros trabajos no son actividades particulares, ni formas económicas diferentes a las del resto del entorno, hace referencia a la cons- 
trucción social que les ha determinado, es así como una actividad puede ser fundamental en un momento determinado de la historia para luego ser considerada un hacer o un trabajo no reconocido. El caso de los chatarreros es claro, y se pueden complementar las comparaciones internacionales, (Medina, 2007; Samson, 2009; Scheinberg, 2012; GAIA, 2012) con nuevas ciudades y diferentes formas de organización de chatarreros.

La experiencia teórica latinoamericana ha sido básicamente ignorada en el contexto europeo, solamente es necesario ver las bibliografías de grandes autores sobre el tema y existe una exclusión sistemática, a excepción de un par de referencias (Sassen, Hart). Al mismo tiempo, la bibliografía latinoamericana carece de nuevas incorporaciones o la recuperación de viejos autores. Es necesario incorporar autores como Enzo Mingione o Robert Pahl a la discusión sobre el trabajo informal.

Los otros trabajos como bien lo indica la bibliografía, y como lo ejemplifican los estudios de caso, son una fuente determinante en el panorama político actual. Las capacidades de subversión y la transversalidad de sus reclamos superan a las clásicas reivindicaciones sindicales, las luchas de chatarreros en Barcelona estuvieron ligadas a asociaciones de vecinos y movimientos sociales en contra de la discriminación. Al igual que lo están los vendedores ambulantes de la ciudad junto a reivindicaciones por el uso libre del espacio público, la crítica al modelo turístico concentrativo de la ciudad, o luchas antirracistas.

En igual medida, es indispensable el entrecruzamiento de variables para entender mejor estos otros trabajos y a los otros trabajadores. No sólo hace falta entender que el trabajo de los chatarreros está estigmatizado, también es necesario relacionar el trabajo con los grupos específicos que lo llevan a cabo. Sujeto y trabajo hacen parte de la misma relación, Es necesario entender el peso de la necesidad de ingresos, el control institucional, el racismo, la fragmentación del mercado de trabajo en la reproducción de la actividad.

No es necesaria una glorificación del trabajo, sin embargo, es necesario resignificar el trabajo en una época en la que el modo de acumulación dominante excluye a la mayor parte de esfuerzos y transformaciones que hacemos las personas en este sistema. Resignificar el hacer es tener una postura crítica con la simbología que tiene el dinero y la forma en como circula y se distribuye.

Es necesario ampliar las perspectivas a teorías más lejanas geográfica y culturalmente. Al tiempo que adaptar las enseñanzas que existe sobre formas de trabajo no hegemónico, como pueden ser los estudios sobre el campesinado, los neo rurales y las nuevas comunidades de consumo responsable, bancos de tiempo, cooperativismo, etc. 
Polis, Revista Latinoamericana, Volumen 15, $N^{\circ}$ 45, 2016

\section{Nota}

${ }^{1}$ Este artículo hace parte del trabajo investigativo llevado a cabo para la obtención del título de doctor en el Programa de Doctorado en sociología de la Universidad de Barcelona. Utiliza 10 de las entrevistas semi-estructuradas realizadas a chatarreros en 2013, observación, participación en eventos y análisis de prensa y de textos jurídicos sobre la actividad. 


\section{Bibliografía}

Arendt, H. (2009). La condición humana. Barcelona: Paidós.

Arrazola, M., Hevia, J. d., Mauleón, I., \& Sánchez, R. (Enero/Febrero de 2011). Estimación del volumen de economía sumergida en España. Cuadernos de Información Económica(220), p.81-89.

Bauman, Z. (2004). Modernidad líquida.Fondo de Cultura Económica, Buenos Aires.

Bodenheimer, S. J. (Abril-junio de 1970). La ideología del desarrollismo: paradigma supletorio de las ciencias políticas norteamericanas para estudios latinoamericanos. Desarrollo económico, 10(37).

Burda, M., Hamermesh, D. S., \& Weil, P. (2013). Total work and gender: facts and possible explanations. Journal of population economics, p.239261.

Castells, M. (2000). La era de la información (Vol. 1), Alianza Editorial, Madrid.

Castillo, J. J. (1998). A la busqueda del trabajo perdido, Tecnos, Madrid.

Castillo, J. J., \& de la Garza Toledo, E. (2000). Tratado Latinoamerica de Sociología del Trabajo, Fondo de Cultura Económica, México.

Chen, M. (2012). The Informal Economy: Definitions, Theories and Policies, Wiego, Cambridge.

Cimolli, M., Primi, A., \& Pugno, M. (Abril de 2006). Un modelo de bajo crecimiento: la informalidad como restricción estructural. Revista de la CEPAL(88), p.89-107.

Coraggio, J. L. (1998). Economía urbana: La perspectiva popular, AbyaYala, Quito.

Credit Suisse. (2015). Global Wealth Report 2015, Credit Suisse AG, Zurich.

Cussiánovich. (2006). Ensayos sobre infancia: Sujeto de derechos y protagonista, Ifejant, Lima.

de la Garza Toledo, E. (2001). Problemas clásicos y actuales de la crisis del trabajo. En E. de la Garza Toledo, \& J. C. Neffa, El trabajo del futuro, el futuro del trabajo, Clacso, Buenos Aires.

de la Garza Toledo, E. (2011). Problemas conceptuales, relaciones de trabajo y derechos laborales de los trabajadores informales. Revista Internacional de Estadística y Geografía, 2(3), p.5-15. 
de la Garzo Toledo, E. (2011). Trabajo no clásico y acción colectiva (Vol. 1). Plaza y Valdes, México DF.

De Lucio, V. (Febrero de 2004). efdeportes.com. Recuperado el 30 de Agosto de 2013, de http://www.efdeportes.com/efd69/aerob.htm

de Soto, H., Ghersi, E., \& Ghibellini, M. (1987). El otro sendero: la revolución informal, Oveja Negra, Lima

de Sousa Santos, B. (2011). Producir para vivir: Los caminos de la producción no capitalista, Fondo de Cultura Económica, México DF.

Escobar, A. (2010). Una minga para el postdesarrollo:lugar, medio ambiente y movimientos sociales en las transformaciones globales. Universidad Nacional Mayor de San Marcos, Lima.

GAIA. (2012). On the Road to Zero Waste: Successes and Lessons From Arround the World. GAIA.

Granovetter, M. (1973). The strength of weak ties. American Journal of Sociology, 78(6), p.1360-1380.

Hart, K. (1973). Informal Income Opportunities and Urban Employment in Ghana. The Journal of Modern African Estudies, p.61-89.

Hart, K. (2000). The Memory Banck: Money in a Unequal World, Profile Books, Londres.

Lazarte, R. (Abril-junio de 2000). El “sector informal” una revisión conceptual bibliográfica. (IIEc-UNAM, Ed.) Problemas del desarrollo, 31(121), p. 35-62.

Lopez Chicharro, J., \& Fernández Vaquero, A. (2006). Fisiología del esfuerzo y el deporte ( $3^{\mathrm{a}}$ ed.), Médica Panamericana, Madrid.

Martinez Veiga, U. (1989). El otro desempleo: La economía sumergida. Cuadernos de antropología, 10, Altamira Press, Lanham.

Mendoza, I. (2014). Afrikalando in the gueto: Prácticas de reciclaje urbano en Barcelona. Tesis Máster, Universidad de Barcelona, Máster enAntropología i etnografía.

Mingione, E. (1994). Las Sociedades Fragmentadas: Una Sociología de la Vida Económica Más Allá Del Paradigma Del Mercado, Ministerio de Trabajo y Seguridad Social, Madrid.

Mires, F. (1991). Una pregunta que es también un tema: ¿existe el sector informal? En J. Scahtan, D. Paas, \& Á. Orsati, El Sector informal en Ameìrica Latina : una seleccioìn de perspectivas analiiticas, Centro de Investigacioìn 
y Docencia Econoìmicas, México DF.

Moreno, A. (2001). Economía popular y desarrollo humano, Abya-Yala, Quito.

Neuwirth, R. (2011). Stealth of nations: the global rise of the informal economy, Phanteon Books, New York.

OIT. (2002). El trabajo decente y la economía informal, Organización Internacional del Trabajo, Ginebra.

Pahl, R. (1991). Divisiones del trabajo, Ministerio de Trabajo y Seguridad Social, Madrid.

Pfau-Effinger, B., Flaquer, L., \& Jensen, P. H. (2009). Formal and Informal Work. The Hidden Work Regime in Europe, Routledge, Taylor \& Francis, New York.

Polanyi, K. (1989). La Gran transformación : crítica del liberalismo económico, La Piqueta, Madrid.

Porras, J. (2013). Mineria urbana a cielo abierto: Los chatarreros subsaharianos en Barcelona. Tesis de Máster, Universidad de Barcelona, Máster en Investigación en Sociología.

Portes, A., \& Zhou, M. (Abril de 1996). Self-Employment and the Earnings of Immigrants. American Sociological Review, 61(2), p.219-230.

Ramírez Guerrero, J. (2003). Capacitación laboral para el sector informal en Colombia. Documento de Trabajo.

Reichman, J., Madorrán, C., \& Echavarría, M. (2013). „Peligro! Hombres trabajando. El trabajo en la era de la crisis ecológico-social, Los Libros de la Catarata, Madrid.

Reskin, B. (2012). The Race Discrimination System. The Annual Review of Sociology, p.17-35.

Samson, M. (2009). Rechazando a ser excluidos: la organización de los recicladores en el mundo, Wiego, Cambridge.

Sassen, S. (1988). New York City's Informal Economy. Volume IV. 1988-89 - Conference on Comparative Ethnicity: The Conference Papers, June 1988, University of California, Los Angeles.

Scheinberg, A. (2012). Informal Sector Integration and High Performance Recycling: Evidence from 20 Cities., Wiego, Cambridge.

Schneider, F. (2005). Shadow economies around the world: what do we 
really know? European Journal of Political Economy(21), p.598-642.

Stovel, K., \& Shaw, L. (2012). Brokerage. The Annual Review of Sociology, 38, p. 139-158.

Torns, T., \& Recio, C. (2012). La desigualdad de género en el mercado de trabajo: Entre la continuidad y la transformación. Revista de Economía Crítica(14), p.178-202.

Wacquant, L. (2010). Castigar a los pobres: El gobierno neoliberal de la inseguridad social, Gesida, Barcelona.

Wallerstein, I. (2013). Structural crisis, or why capitalist may no longer find capitalism rewarding. En I. Wallerstein, C. Randall, M. Michael, G. Derlugian, \& C. Calhoun, Does capitalisn have a future?(págs. 9-37), Oxford University Press, New York. 